\title{
Incidence of infective valve endocarditis as after antibiotic prophylaxis guidelines changed-there is no change
}

\author{
Efstratios I. Charitos ${ }^{1}$, Jan-Malte Sinning ${ }^{2}$ \\ ${ }^{1}$ Department of Cardiac Surgery, ${ }^{2}$ Department of Cardiology, University of Bonn, Bonn, Germany \\ Correspondence to: Efstratios I. Charitos, MD, PhD. Department of Cardiac Surgery, University of Bonn, Venusberg Campus 1, 53127, Bonn, \\ Germany. Email: efstratios.charitos@gmail.com.
}

Submitted Aug 25, 2019. Accepted for publication Sep 06, 2019.

doi: 10.21037/acs.2019.09.01

View this article at: http://dx.doi.org/10.21037/acs.2019.09.01

"It ain't what you don't know that gets you into trouble. It's what you know for sure that just ain't so."

—Mark Twain

Infective endocarditis (IE) is a rare disease, however, it is associated with significant morbidity and mortality. One of the most recognizable portals of entry for pathogens causing IE is the oral cavity, identified almost 100 years ago. Transient bacteremia has been demonstrated in several studies, and the seemingly obvious causal relationship to the development of IE has been established. Since then, advances in risk stratification have identified patients at high, moderate, or low risk for endocarditis and for over 50 years antibiotic prophylaxis (AP) has been recommended to protect vulnerable individuals from developing IE.

This seemingly straightforward causal relationship, however, has not withstood scientific vigor. Beginning with the 2007 revisions, the ESC and AHA guidelines have questioned both the necessity and effectiveness for AP for the prevention of IE in all but a small subgroup of patients at the highest risk for developing IE. The NICE guidelines from the UK had abolished AP for all patients in 2008, and the 2016 minor revision does not routinely recommend AP. This change has been based on several facts. The causal relationship between transient bacteremia and IE has not been proven. Although AP may reduce transient bacteremia, it fails to prevent it in up to $50 \%$ of cases. Several everyday activities (chewing) and procedures (tooth brushing and dental hygiene) produce transient bacteremia to a similar extent. There is no compelling evidence that respiratory tract, gastrointestinal, genitourinary, dermatological or musculoskeletal procedures on non-infected tissue can cause IE. Several population studies have questioned the role of dental interventions as a risk factor for IE, and widespread and indiscriminate AP can contribute to the burden of antibiotic resistance. Although dental procedures are not the sole indication for AP prescription, it is by far the most common (1).

Since the paradigm change in the guidelines regarding AP starting in 2007, several studies have tried to investigate the effect of this shift in the incidence of endocarditis. Some studies failed to show an increase in the incidence of IE after the introduction of the new guidelines (2-5), whereas other studies have raised concerns regarding increasing incidence of IE (6-9). In the face of a lack of randomized controlled trials regarding AP, all previously mentioned studies will inevitably have methodological errors which hinder drawing scientifically rigorous conclusions. Unsurprisingly, various and contradicting arguments, counter arguments and conclusions from these studies can be drawn. The failure to detect an increase in the incidence of IE after the guideline change might be due to methodological drawbacks of the studies. The increased incidence of IE reported by other studies might be attributed to issues on statistical modelling, methodological concern, changes in population demographics, increase in interventions and cardiac implants, failure to detect the real incidence of IE or to report it, or failure to code specific pathogens may help draw conclusions about the effectiveness of AP.

Although these studies have led to discussions in the literature (5), it is important not to lose the scope of the original questions. Is AP effective in preventing transient bacteremia and are these events causal for development of IE? Probably not. Can an increase in the incidence of IE provide a sound steppingstone for the widespread prescription of AP? Probably not. It should now be clear 
to everyone that the incidence of IE will increase in the future. The aging population, the significant incidence of valvular disease with advanced age, the exponential growth of cardiac implants-especially in the elderly or sick-the increased survival of patients with significant comorbidities requiring cardiac implants, interventions or hospitalization as well as advances in awareness and diagnostic modalities make the increase in IE incidence almost inevitable. The change in pathogens observed in IE in the last years also provides an argument for this shift. Several studies have shown that especially in frail and sick patients, enterococcal and staphylococcal pathogens are the most frequent (10). AP will have little, if any effect in preventing IE due to these pathogens and entry paths.

If we still have significant questions on both the necessity and effectiveness of AP, and if we still lack randomized controlled trials regarding AP, does this justify a leap of faith for the widespread prescription of AP? Probably not. If we go down the road of widespread AP prescription, where do we draw the line? Male individuals, older individuals, patients with significant comorbidities, hospitalized patients are all at an increased risk for IE. The population at risk only gets bigger down this road, without hard evidence to support such a stance.

While one could argue that the 2008 NICE guidelines were perhaps too strict, the ESC and AHA guidelines take a sensible approach. They both recognize that there is a significant lack of evidence regarding $\mathrm{AP}$ and restrict its widespread use, while still recommending AP in patients with the highest risk for IE, in which an IE episode carries probably the highest morbidity and mortality. They also note the importance of flagging patients with prosthetic cardiac valves, patients with previous episodes of IE and patients with significant congenital heart disease. Whether AP is necessary in this population is still unproven, however, given the scarcity of quality data on this topic, it seems a sensible approach. In a constantly changing landscape of cardiovascular diseases, changing patient characteristics and exponential growth of interventions and cardiac implants, the restriction of AP based on the available evidence seems a step in the right direction with no effect on the incidence of IE.

However, as physicians, the burden of proving the effectiveness and necessity of AP in preventing IE still weighs heavily on our shoulders.

\section{Acknowledgments}

None.

\section{Footnote}

Conflicts of Interest: The authors have no conflicts of interest to declare.

\section{References}

1. Cahill TJ, Baddour LM, Habib G, et al. Challenges in Infective Endocarditis. J Am Coll Cardiol 2017;69:325-44.

2. Bikdeli B, Wang Y, Kim N, et al. Trends in hospitalization rates and outcomes of endocarditis among Medicare beneficiaries. J Am Coll Cardiol 2013;62:2217-26.

3. DeSimone DC, Tleyjeh IM, Correa de Sa DD, et al. Temporal trends in infective endocarditis epidemiology from 2007 to 2013 in Olmsted County, MN. Am Heart J 2015;170:830-6.

4. Duval X, Delahaye F, Alla F, et al. Temporal trends in infective endocarditis in the context of prophylaxis guideline modifications: three successive population-based surveys. J Am Coll Cardiol 2012;59:1968-76.

5. Mackie AS, Liu W, Savu A, et al. Infective Endocarditis Hospitalizations Before and After the 2007 American Heart Association Prophylaxis Guidelines. Lancet 2015;385:1219-28.

6. Dayer MJ, Jones S, Prendergast B, et al. Incidence of infective endocarditis in England, 2000-13: a secular trend, interrupted time-series analysis. Lancet Lond Engl 2015;385:1219-28.

7. Pant S, Patel NJ, Deshmukh A, et al. Trends in infective endocarditis incidence, microbiology, and valve replacement in the United States from 2000 to 2011. J Am Coll Cardiol 2015;65:2070-6.

8. Keller K, von Bardeleben RS, Ostad MA, et al. Temporal Trends in the Prevalence of Infective Endocarditis in Germany Between 2005 and 2014. Am J Cardiol 2017;119:317-22.

9. van den Brink FS, Swaans MJ, Hoogendijk MG, et al. Increased incidence of infective endocarditis after the 2009 European Society of Cardiology guideline update: a nationwide study in the Netherlands. Eur Heart J Qual Care Clin Outcomes 2017;3:141-7.

10. Amat-Santos IJ, Ribeiro HB, Urena M, et al. Prosthetic valve endocarditis after transcatheter valve replacement: a systematic review. JACC Cardiovasc Interv 2015;8:334-46.

Cite this article as: Charitos EI, Sinning JM. Incidence of infective valve endocarditis as after antibiotic prophylaxis guidelines changed-there is no change. Ann Cardiothorac Surg 2019;8(6):681-682. doi: 10.21037/acs.2019.09.01 